\title{
Adrenal myelolipoma with hyperandrogenemia and schizophrenia
}

This article was published in the following Dove Press journal: Cancer Management and Research

\author{
Ningning Liu ${ }^{1,2, *}$ \\ Wenhua Zhang ${ }^{3, *}$ \\ Jiangshan $\operatorname{Tan}^{1,4}$ \\ Jiaxin Zhou' \\ Xiaopeng $\mathrm{Yu}^{5}$ \\ Ningxin $\operatorname{Ren}^{5}$ \\ Zhiqing Fang ${ }^{3}$ \\ Wei Jiao ${ }^{3}$ \\ Yidong Fan ${ }^{3}$ \\ Cheng Liu ${ }^{6}$
}

'School of Medicine, Shandong University, Jinan, China; ${ }^{2}$ Institute of Mental Health, Peking University, The Sixth Hospital, Peking University, Beijing, China; ${ }^{3}$ Department of Urology, Qilu Hospital, Shandong University, Jinan, China; ${ }^{4}$ State Key Laboratory of Cardiovascular Disease, FuWai Hospital, Peking Union Medical College and Chinese Academy Medical Science, Beijing, China; ${ }^{5}$ School of Basic Medicine Sciences, Shandong University, Jinan, China; ${ }^{6}$ Department of Urology, Peking University Third Hospital, Beijing, China

*These authors contributed equally to this work
Correspondence: Yidong Fan; Wei Jiao Department of Urology, Qilu Hospital, Shandong University, Wenhua Xilu 107, 250012 Jinan, China

Email qlfanyidong@163.com; jiaowei3929@।63.com

\begin{abstract}
Adrenal myelolipoma with hyperandrogenemia is extremely rare. We report a case of a 26-year-old Chinese female with schizophrenia, who presented with a hormonally active tumor causing hyperandrogenemia. The mass was found by computerized tomography when she had her gynecologic examination for secondary amenorrhea, and it was confirmed to be an adrenal myelolipoma after a histopathological study. She was referred for a left adrenal laparoscopic excision, and the size of adrenal myelolipoma was found to be more than $10 \mathrm{~cm}$. We report this case because large adrenal myelolipomas with hyperandrogenemia and schizophrenia are rare, and adrenal myelolipoma associated with hyperandrogenemia might be determined by the enzymes involved in the production of hormones.
\end{abstract}

Keywords: adrenal, hyperandrogenemia, myelolipoma, schizophrenia

\section{Introduction}

Adrenal myelolipoma is infrequently encountered nonfunctioning benign tumor of unknown etiology. In some cases, it presents endocrine disorders, such as Cushing's syndrome, Conn's syndrome, and congenital adrenal hyperplasia. ${ }^{1}$ But few cases of virilization have been reported. To our knowledge, this kind of case is very rare, and there are only three related documented case reports in the English literature. Except for virilization and other common symptoms, this kind of adrenal myelolipoma often accompanies other abnormalities such as type- 2 diabetes mellitus, acanthosis nigricans, and growth retardation. We report one case with schizophrenia, and we hypothesize that it is associated with her tumor.

\section{Case report}

A 26-year-old woman with schizophrenia was admitted to our hospital for a left adrenal mass found when she had her gynecologic examination for secondary amenorrhea.

The patient was a hepatitis B positive female with a history of schizophrenia for more than 10 years, who exhibited male characteristics (including hirsutism and menoxenia) but with no lumbago. At the age of 13 years, the patient began to menstruate, but the cycles were rather irregular. Six years ago, her menstruation cycle became normal because she was consuming ethinylestradiol and cyproterone acetate tablets. In addition, she has been taking antipsychotic drugs for more than 10 years.

On a through laboratory examination, the patient had a high level of plasma testosterone (328.79 ng/mL). Complete blood count revealed increasing leukocytes 
$(47.00 / \mu \mathrm{L})$, thrombocytocrit $(0.41 \%)$, and epithelia $(33.5 / \mu \mathrm{L})$. The level of plasma cortisol aldosterone, basal plasma renin activity, and angiotensin II showed no obvious abnormality. Abdominal sonography showed that there was a well-defined hyperechoic heterogeneous mass of $11.0 \times 10.1 \times 10.1$ between the left adrenal gland and spleen. Computerized tomography scan of the abdomen with oral and intravenous contrast showed a big nonhomogeneous mass pushing the left kidney down in the left upper quadrant. It measured approximately $11.6 \times 10.0 \mathrm{~cm}$ with multiple septa which showed slight enhancement in arterial phase and moderate enhancement in venous phase, so did the solid component of the tumor. This mass also had a vaguely defined border with the tail of the pancreas and spleen. Besides, a low-density lesion could be seen in the left lobe of the liver (Figures 1 and 2).

The left adrenal mass specimen measured $13.5 \times 10.5 \times 6.5 \mathrm{~cm}$. A mixture of mature adipose tissue and bone marrow elements could be seen in the low-power histological picture (Figure 3). Immunohistochemical staining was performed for Syn, Ck, Hmb45, Melan-A, Hmb45, and cgA (Figure 3).

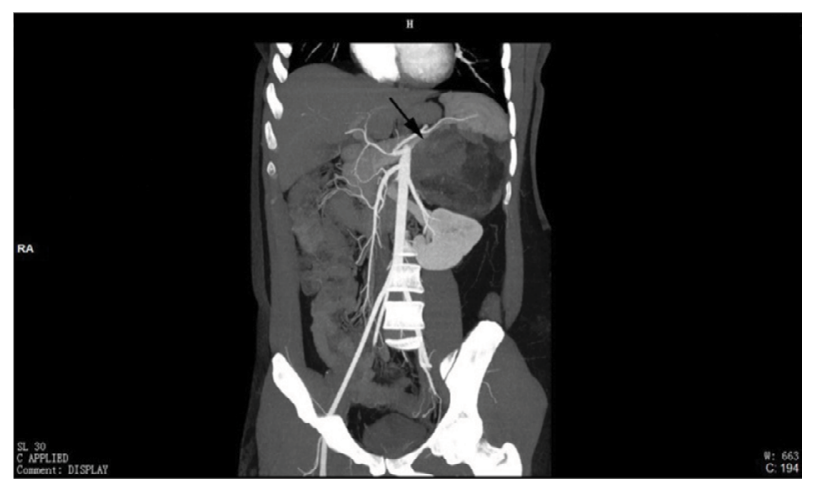

Figure I Coronal image from contrast-enhanced CT showing a left adrenal mass; left kidney is displaced inferiorly by the mass.

Abbreviation: CT, computerized tomography.

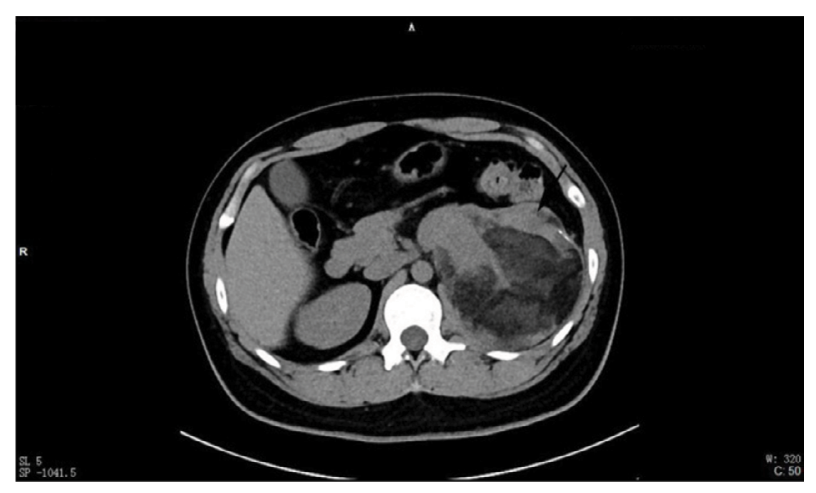

Figure 2 Axial plane showing adipose tissue and septations. Notes: Tiny foci of calcifications are also visible in the lateral wall of the mass.

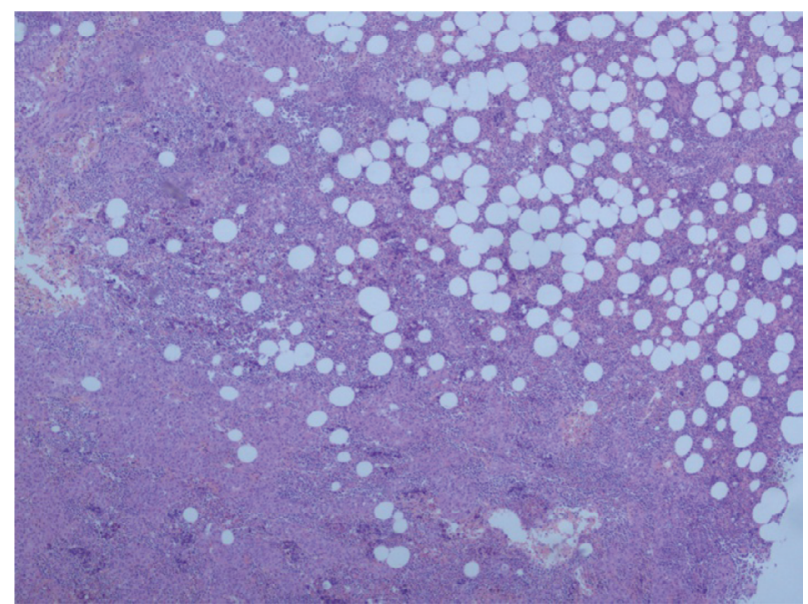

Figure 3 Low-power histological image showing a mixture of mature adipose tissue and bone marrow elements $(\times 10)$.

The patient was referred for a left adrenal laparoscopic excision, and the postoperative hospital course was uneventful. She recovered quickly, and the level of plasma testosterone was $0.68 \mathrm{ng} / \mathrm{mL}$ (Figure 4). Her menstruation soon became regular and endocrine examinations returned to normal.

The patient's family gave their written informed consent for all or any part of this material to appear in this paper and all editions of Cancer Management and Research, and any other works or products, in any form or medium.

\section{Discussion}

Myelolipoma is a rare and benign neoplasm which was first described by Gierkein ${ }^{2}$ and named by Oberling in 1929. In the previously reported cases, endocrine disorders, such as Cushing's syndrome, Conn's syndrome, and congenital adrenal hyperplasia as a result of 17 a-hydroxylase or 21a-hydroxylase deficiency, ${ }^{1}$ were found to be associated with functioning tumors, myelolipoma with virilization was very rare, especially with such a large mass.

Most myelolipomas are asymptomatic and discovered incidentally on abdominal imaging, such as ultrasonography and computerized tomography, for some other indications. ${ }^{1}$ Some may present with abdominal pain because of huge size or hemorrhage or necrosis within tumor. The patient in the current case had no abdominal pain or other related discomfort even though the size of adrenal myelolipoma was more than $10 \mathrm{~cm}$. That was why she did not find that her virilization was associated with the adrenal tumor until she underwent a gynecologic examination for secondary amenorrhea.

The etiology of adrenal myelolipoma is still unknown. Past researches found adrenal myelolipoma is associated with long periods of elevated ACTH. In addition, chronic stressful conditions such as diabetes mellitus, hypertension, 


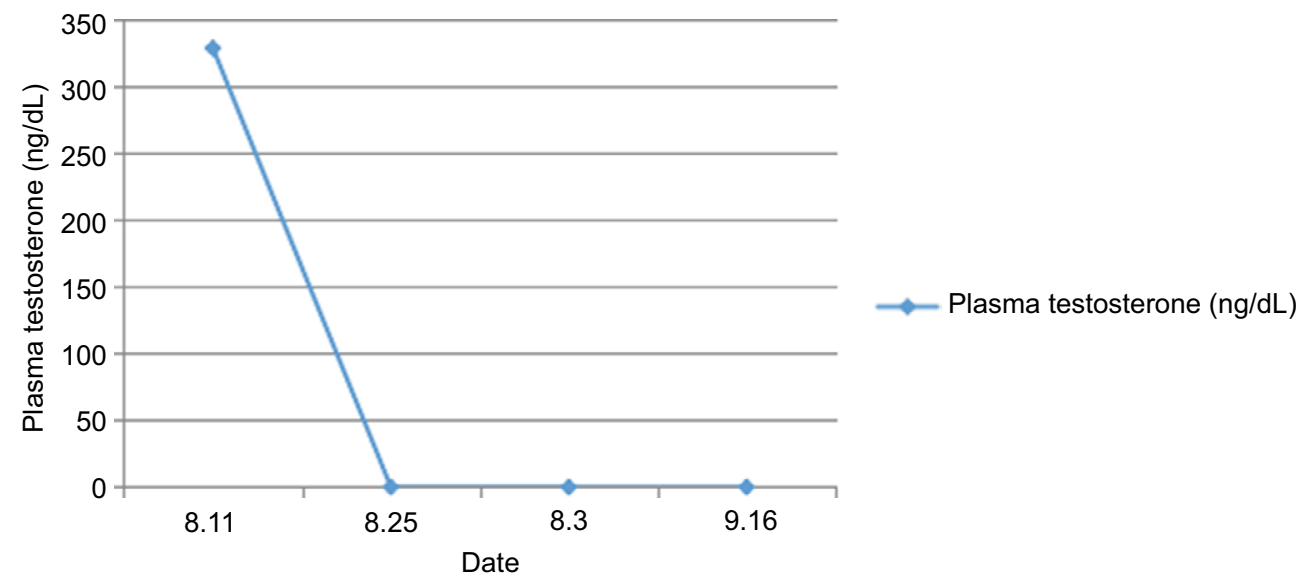

Figure 4 The patient was referred for a left adrenal laparoscopic excision on August 22, 2016. Notes: After the surgery, her plasma testosterone level was back to normal.

Table I Adrenal myelolipoma associated with hyperandrogenemia

\begin{tabular}{llllll}
\hline Study & Size & $\begin{array}{l}\text { Age } \\
\text { (years) }\end{array}$ & $\begin{array}{l}\text { Left/ } \\
\text { right }\end{array}$ & Cortisol & $\begin{array}{l}\text { Lumbar } \\
\text { region }\end{array}$ \\
\hline $\begin{array}{l}\text { Moskovitz et al, }{ }^{10} \\
\text { I } 989\end{array}$ & $3.5 \times 3 \times 2$ & 34 & Right & $/$ & $/$ \\
$\begin{array}{l}\text { Tasnim Ahsan } \\
\text { et al, }{ }^{\prime \prime} 2010\end{array}$ & $5.5 \times 4.5$ & 25 & Left & $/$ & Yes \\
$\begin{array}{l}\text { Su et al, }{ }^{4} 2012 \\
\text { Our case }\end{array}$ & $\begin{array}{l}7 \times 5 \\
13.5 \times 10 \times 6.5\end{array}$ & 26 & Left & Normal & No \\
\hline
\end{tabular}

Note: "l" indicates that the study gave no information about this factor.

obesity, chronic inflammatory processes, and malignancy are also be observed in patients with adrenal myelolipoma. ${ }^{3}$ On examination, the body mass index of the patient in the current case was $27.89 \mathrm{~kg} / \mathrm{m}^{2}$, which is indicative of obesity. But, she has neither hypertension nor diabetes.

Adrenal myelolipoma associated with hyperandrogenemia is much rarer (Table 1). One report hypothesizes that it might be determined by the aforementioned multiple factors, extrinsic compression of the large tumor, and the enzymes involved in the production of hormones, such as melamin-A, ${ }^{4}$ In our case, Melan-A immunohistochemical staining was also positive. In addition, positive Syn staining indicated that the mass had neuroendocrine function, which could explain the elevated levels of androgen. In addition, extra-adrenal myelolipoma with virilization and Cushing's syndrome has also been reported before in patients whose immunohistochemistry showed that the mass was positive for calretinin, Melan-A, and Syn. ${ }^{5}$

This patient had a history of schizophrenia for more than 9 years and has been taking antipsychotic drugs for more than 5 years. Schizophrenia is a brain disorder that affects how people think, feel, and perceive. People with schizophrenia can experience both hyper- and hypofunction of the hypothalamic-pituitary-adrenal axis, ${ }^{6}$ as shown by a new study. In addition, another study shows that patients with schizotypal disorder, compared with healthy control subjects, have an enlarged pituitary volume. ${ }^{7}$ So, we hypothesize that her schizophrenia may have caused the adrenal myelolipoma. Or, her schizophrenia may have been caused by the adrenal myelolipoma.

People hold different opinion regarding the size criteria for adrenal myelolipomas as an indication for surgical resection. Previously, laparoscopic approach was not considered a good choice when the adrenal tumor size exceeded 5-6 cm. But through the recent advancement of diagnostic imaging methods, some surgeons demonstrated that laparoscopic adrenalectomy could be used in giant adrenal myelolipoma. ${ }^{8} \mathrm{~A}$ tumor with a large size, $15 \mathrm{~cm}$ at its longest dimension, has been resected by the laparoscopic approach recently. ${ }^{9}$ The tumor mass in our patient was removed with laparoscopic resection, and her postoperative hospital course was uneventful.

Adrenal myelolipoma with virilization is very rare. We reported here a case with schizophrenia, and we hypothesize that this may explain the pathogenesis of the present case, or, vice versa, the adrenal myelolipoma could have caused her schizophrenia. In addition, this case supports the hypothesis that adrenal myelolipoma associated with hyperandrogenemia might be determined by the enzymes involved in the production of hormones.

\section{Limitations}

Disadvantage: It is only our conjecture that her schizophrenia is related to this tumor. Long-term follow-up should been done to observe the mental disorder and adrenal gland varieties of these patients by using imaging methods. If it is possible, genetic tests are likely to be helpful too. 


\section{Acknowledgments}

The study was funded by the National Natural Science Foundation of China (No. 81711530048, No. 81372765, No. 81572515 , No. 81472395 , and No. 81672522); Shandong Provincial Natural Science Foundation, China (No. ZR2011HM055, No. ZR2014HQ035, and No. BS2014YY036); and the Research Fund of Shandong University Qilu Hospital (No. 2014QLKY16).

\section{Disclosure}

The authors report no conflicts of interest in this work.

\section{References}

1. Hisamatsu H, Sakai H, Tsuda S, Shigematsu K, Kanetake H. Combined adrenal adenoma and myelolipoma in a patient with Cushing's syndrome: case report and review of the literature. Int J Urol. 2004;11:416-418.

2. Gierke E. Uber Knochenmarksgewebe in der Nebenniere. Beitr Pathol Anat. 1905:26.
3. Gee WF, Chikos PM, Greaves JP, Ikemoto N, Tremann JA. Adrenal myelolipoma. Urology. 1975;5:562-566.

4. Su HC, Huang X, Dai J, Sun FK. Adrenal myelolipoma associated with hyperandrogenemia. Int J Urol. 2012;19:1026-1028.

5. Liu W, Chen W, He X. An unusual cause of cushing's syndrome and virilization. Gastroenterology. 2015;149:e5-e6.

6. Bradley AJ, Dinan TG. A systematic review of hypothalamic-pituitaryadrenal axis function in schizophrenia: implications for mortality. J Psychopharmacol. 2010;24:91-118.

7. Takahashi T, Suzuki M, Velakoulis D et al. Increased pituitary volume in schizophrenia spectrum disorders. Schizophr Res. 2009;108:114-121.

8. Park BH, Lee SL, Seo KJ et al. Laparoscopic hand-assisted adrenal sparing surgery for a giant adrenal myelolipoma: A case report. Int Surg. Epub 2015 May 19.

9. Chaudhary R, Deshmukh A, Singh K, Biswas R. Is size really a contraindication for laparoscopic resection of giant adrenal myelolipomas? BMJ Case Rep. 2016;2016.

10. Moskovitz B, Bolkier M, Katz Y, Arieh YB, Levin DR. Adrenal myelolipoma simulating virilizing adrenal tumor. Urologia Internationalis. 1989;44(1):53-55.

11. Ahsan T, Kanwal S, Banu Z, Jabeen R. Virilization with adrenal myelolipoma, adrenal hyperplasia, and fibroadenoma of breast. J Coll Physicians Surg Pak. 2010;20(12):819-821.
Cancer Management and Research

\section{Publish your work in this journal}

Cancer Management and Research is an international, peer-reviewed open access journal focusing on cancer research and the optimal use of preventative and integrated treatment interventions to achieve improved outcomes, enhanced survival and quality of life for the cancer patient. The manuscript management system is completely online and includes
Dovepress

a very quick and fair peer-review system, which is all easy to use. Visit $\mathrm{http}: / / \mathrm{www}$.dovepress.com/testimonials.php to read real quotes from published authors. 